\title{
BMJ Global Health Effects of community health worker interventions on socioeconomic inequities in maternal and newborn health in low-income and middle- income countries: a mixed-methods systematic review
}

To cite: Blanchard AK, Prost A, Houweling TAJ. Effects of community health worker interventions on socioeconomic inequities in maternal and newborn health in low-income and middle-income countries: a mixed-methods systematic review. BMJ Global Health 2019;4:e001308. doi:10.1136/ bmjgh-2018-001308

Handling editor Seye Abimbola

- Additional material is published online only. To view please visit the journal online (http://dx.doi.org/10. 1136bmjgh-2018-001308)

Received 14 November 2018 Revised 30 April 2019 Accepted 4 May 2019

Check for updates

(c) Author(s) (or their employer(s)) 2019. Re-use permitted under CC BY-NC. No commercial re-use. See rights and permissions. Published by BMJ.

${ }^{1}$ Institute for Global Health, University College London, London, UK

${ }^{2}$ Department of Public Health, Erasmus MC, Rotterdam, The Netherlands

Correspondence to Andrea Katryn Blanchard; andrea.blanchard.15@ucl.ac.uk

\section{ABSTRACT}

Introduction Community health worker (CHW) interventions are promoted to improve maternal and newborn health in low-income and middle-income countries. We reviewed the evidence on their effectiveness in reducing socioeconomic inequities in maternal and newborn health outcomes, how they achieve these effects, and contextual processes that shape these effects. Methods We conducted a mixed-methods systematic review of quantitative and qualitative studies published between 1996 and 2017 in Medline, Embase, Web of

Science and Scopus databases. We included studies examining the effects of CHW interventions in low-income and middle-income countries on maternal and newborn health outcomes across socioeconomic groups (wealth, occupation, education, class, caste or tribe and religion). We then conducted a narrative synthesis of evidence. Results We identified 1919 articles, of which 22 met the inclusion criteria. CHWs facilitated four types of interventions: home visits, community-based groups, cash transfers or combinations of these. Four studies found that CHWs providing home visits or facilitating women's groups had equitable coverage. Four others found that home visits and cash transfer interventions had inequitable coverage. Five studies reported equitable effects of $\mathrm{CHW}$ interventions on antenatal care, skilled birth attendance and/or essential newborn care. One study found that a CHW home visit intervention did not reduce wealth inequities in skilled birth attendance. A study of women's groups reported greater reductions in neonatal mortality among lower compared with higher socioeconomic groups. Equity was most improved when CHWs had relevant support for assisting women to improve health practices and access health care within community contexts. Conclusion While current evidence remains limited, particularly for mortality, existing studies suggest that CHW interventions involving home visits, cash transfers, participatory women's groups or multiple components can improve equity in maternal and newborn health. Future mixed-methods research should explore intervention strategies and contextual processes shaping such effects on equity to optimise these efforts.

\section{Key questions}

What is already known?

- Systematic reviews have found that interventions with community health workers (CHWs) can improve maternal and newborn health in low-income and middle-income countries.

- Little is known about whether, how and in what contexts they reduce socioeconomic inequities in maternal and newborn health.

What are the new findings?

- We found that CHWs conducting home visits, facilitating community-based groups or enabling cash transfers had some success in reducing inequities in intervention coverage, behaviours and in a few studies, neonatal mortality across wealth, education and caste groups.

- Equity was most readily achieved when CHWs were well supported to assist families in decision-making and overcome barriers to accessing health services.

What do the new findings imply?

- Monitoring and evaluation must focus on whether equity in coverage follows through to equity in outcomes across the continuum of care for pregnancy delivery and the postnatal period.

- Research and practitioners should seek to better understand and address contextual processes that may otherwise undermine the ability of interventions to reduce inequities in maternal and newborn health between various socioeconomic groups.

\section{INTRODUCTION}

The Sustainable Development Goals have placed renewed emphasis on improving maternal and newborn health, calling for reductions in the global maternal mortality ratio (MMR) and neonatal mortality rate (NMR) to 70 per 100,000 live births and 12 
per 1000 live births, respectively, by $2030 .{ }^{1}$ Maternal and newborn deaths continue to be highest among the most socioeconomically disadvantaged families globally. ${ }^{2}{ }^{3}$ Addressing these inequities is an ethical imperative and essential strategy for achieving overall improvements in survival. Reducing the NMR in the poorest wealth quintile to the level of the wealthiest quintile in low-income and middle-income countries (LMICs) could prevent nearly a quarter of neonatal deaths annually. ${ }^{2}$

Community health workers (CHWs) may have great potential to reduce inequities in maternal and neonatal mortality between socioeconomic groups based on wealth, occupation, education, class, caste, tribe or religion. They have long been positioned at the frontlines in many countries' efforts towards ensuring primary health care. They are also seen as central to achieving universal health coverage in line with the third Sustainable Development Goal. ${ }^{45}$ The WHO has defined CHWs as people who live and work in their communities, are selected by these communities and accountable to them, and may or may not be part of the health system. ${ }^{467}$ CHWs can include trained lay workers, health volunteers, community health agents, traditional births attendants and community midwives. ${ }^{8}$ A systematic review found that interventions with CHWs can improve birth outcomes in LMICs. ${ }^{9}$

What do we know about the effects of CHW interventions on inequities in maternal and newborn health $(\mathrm{MNH})$ and mortality in LMICs? Yuan $e t$ als systematic review identified three studies from Bangladesh showing that interventions where CHWs promoted immunisation services and made home visits improved equity in immunisation coverage between families with lower and higher education, and antenatal care and skilled birth attendance at home between wealth groups. However, these studies did not examine effects on maternal and neonatal mortality. ${ }^{10}$ McCollum et al reviewed evidence on access, uptake and quality of CHW programmes for maternal and newborn health, and found that improvements in the coverage of CHW services were equitable across socioeconomic groups in 3 out of 26 studies. ${ }^{11}$ They did not describe effects on equity in maternal and neonatal behavioural or mortality outcomes. ${ }^{11}$ Another review looked at the effects of community-based primary health care programmes on maternal, newborn and child health. ${ }^{12}$ It found that these programmes improved coverage of $\mathrm{CHW}$ home visits and essential newborn care equitably among poorer and less poor households. ${ }^{12}$

No systematic review has explicitly examined the effects of CHW interventions on inequities from intervention coverage to behavioural and mortality outcomes across the MNH continuum of care in LMICs. In addition, no systematic review has ever focused on the intervention strategies through which these interventions may achieve their equitable effects or the contextual processes that shape these effects. ${ }^{13} 14$ Therefore, we conducted a mixed-methods systematic review to answer the following questions: What are the effects of $\mathrm{CHW}$ interventions on equity in maternal and newborn health in LMICs, how do they achieve these effects, and in what contexts?

\section{METHODS}

\section{Mixed-methods systematic review}

Traditional systematic review methods are not always well suited to consider how complex interventions achieve their effects. ${ }^{15}$ We chose to use a mixed-methods review that could include varied study designs to gather evidence on what works, as well as how and in what contexts. ${ }^{1617}$ A mixed-methods review is apt for fulfilling our aim of understanding effects on equity across LMIC contexts where CHW interventions have relied on different intervention strategies to improve maternal and newborn health. ${ }^{15}$ Here, we consider intervention strategies to be the intentional elements of intervention design that seek to improve MNH. Contextual factors encompass the health care system, social, cultural, economic, political or geographical dynamics in the region of implementation, beyond the specific CHW intervention strategies, that may also shape the effects of interventions on inequities in maternal and newborn health.

\section{Search strategy}

AKB searched the Medline and Embase databases for medical literature, and Web of Science and Scopus for social science literature using the search terms (online supplementary table 1), guided by the Preferred Reporting Items for Systematic Reviews and Meta-Analyses-Equity (PRISMA-E) checklist. ${ }^{18} 19$ The search criteria were organised as follows ${ }^{20-22}$ : first, we used terms to describe different types of interventions implemented by CHWs, such as home visits and women's groups. Second, we included terms related to maternal and newborn health outcomes during pregnancy, delivery and the neonatal period (0-28 days after birth). Third, we included terms to capture different dimensions of socioeconomic position, informed by the PROGRESS framework, including comparisons between different wealth, occupation, class, caste, tribe, religion and literacy or education levels. ${ }^{23}$ Finally, we used terms to describe LMICs. We used the Medical Subject Headings (MeSH) available in each database and manually added terms that were not available.

\section{Inclusion criteria}

We included articles on studies that: (1) examined socioeconomic differences in coverage or effects of CHW interventions in relation to maternal and newborn health outcomes; (2) included participants who were women that recently had a delivery, their families or CHWs in LMICs; (3) used experimental, quasi-experimental, observational or qualitative designs; and (4) were conducted after 1990 and published between 1 January 1996 and 30 August 2017.

AKB and AP screened all abstracts and titles based on the criteria (online supplementary table 2). We then read full texts of all retrieved articles to identify those 
that included equity-related findings. We included articles on studies that compared interventions' coverage or effects on outcomes by socioeconomic position quantitatively using experimental, quasi-experimental and observational designs, as well as those that used qualitative methods to explore intervention strategies and/or contextual factors influencing these effects. We discussed any discrepancies on the articles to include and came to a consensus.

\section{Synthesis of findings}

$\mathrm{AKB}$ and $\mathrm{AP}$ created a data extraction sheet and imported the full text of all articles that met the inclusion criteria into NVivo V.11.0 to code them (online supplementary table 3). We used narrative synthesis that followed an approach with three steps suggested by Petticrew and Roberts $^{15}$ : (1) organising information into local categories; (2) analysing the findings for each of the codes on equity associations with CHW intervention exposure (to address what worked), and intervention strategies or contextual factors; and (3) synthesising findings for all the articles to determine themes on the important strategies through which interventions might have impacted equity in maternal and newborn health (to address how they worked), and the influence of contextual processes (to assess in what contexts they may have worked).

For the first step, after AP and AKB defined the codes in line with the extraction sheet, AKB conducted lineby-line coding. For the second step, AKB used matrix queries to descriptively summarise the results in tables on quantitative associations between intervention exposure and equity in the outcomes by the studies' region, type of CHW intervention, outcome type (coverage, behavioural and mortality) and quality. This was also done for the intervention strategies and contextual factors that were described as related to equity effects in any quantitative or qualitative studies. AP reviewed the descriptions to ensure consistency with the extraction sheet to finalise the summaries. To fulfil step 3, AKB and AP discussed and compared the results on associations, intervention strategies and contextual factors by intervention type and outcome with the original texts with support from TAJH to come to a consensus on the final themes.

\section{Quality appraisal}

Petticrew and Roberts discuss the limitations of adhering strictly to a 'hierarchy of evidence' when appraising the quality of studies on complex health interventions, because relevant evidence can be drawn from studies with a range of different designs. Instead, they favour using a "typology of evidence, ${ }^{15}$ and appraising studies according to criteria relevant to their design. Likewise, we used design-specific criteria to assess internal validity, external validity and trustworthiness of studies, adapted from Petticrew and Roberts (shown in table 1). ${ }^{15}{ }^{24} \mathrm{AP}$ and $\mathrm{AKB}$ agreed on codes for each of the quality criteria, which AKB used in line-by-line coding, as the basis for weighting each study as 'low', 'moderate' or 'high' quality. We described quality as 'low' if there were substantial issues in relation to any of the criteria in table 1 . We described quality as 'moderate' if the study addressed all relevant quality components and discussed strategies to address any issues that weakened the study. Finally, 'high quality' was assigned if the design was clearly described and had no substantive issues relating to the criteria.

\section{Patient and public involvement statement}

No patients or members of the public were involved in this study.

Table 1 Systematic review quality appraisal checklist

\begin{tabular}{ll}
\hline Quality measure & Components \\
Internal validity & Randomisation, blinding and sampling done soundly \\
& Control versus treatment groups with comparable characteristics, drop-outs not systematically \\
& different \\
& Appropriate means of analysis including multiple statistical tests, post hoc or subgroups \\
& Discussion of confounders and how treated \\
& Study design fit-for-purpose-the design matches the research questions being asked and answered \\
& Context described clearly, and attention to effect modification related to intervention \\
External validity & Deplicated in different settings, populations, long duration or among large sample size (with sufficient \\
& power) \\
& Summary of results clear \\
& Clear links between data, interpretation and conclusion \\
& Detailed description of methods and analysis techniques \\
Trustworthiness and transferability \\
(qualitative studies) & Quotes and in-depth, rich description \\
& Sample represents diverse views or across populations and contexts, interventions clearly described \\
& Clarity on the assumptions/ theoretical perspectives/ values or positionality of researchers
\end{tabular}




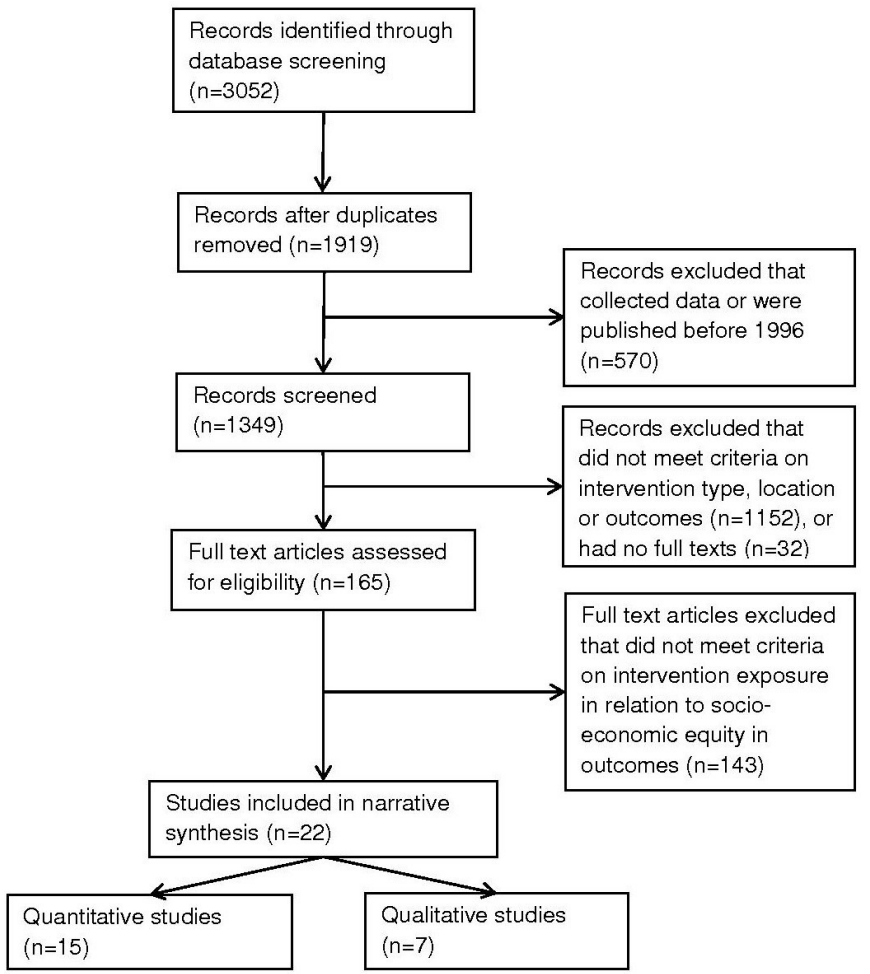

Figure 1 Flow chart depicting the selection of studies.

\section{RESULTS}

The search yielded 1919 unique articles (figure 1). Of these, 165 articles were identified after title and abstract screening, of which 22 met the inclusion criteria after full-text screening.

Online supplementary table 4 shows details of study designs, results of the quality appraisal and an overview of equity findings. The quantitative and qualitative studies investigated interventions in South Asia $(n=15)$, Africa $(n=2)$, Latin America $(n=1)$, Southeast Asia $(n=2)$ or multiple countries $(n=2)$, as shown in figure 2. The interventions described in these articles fell into four broad types: home visits; community-based groups; cash transfers; mixed interventions involving multiple components, such as community meetings, media campaigns or

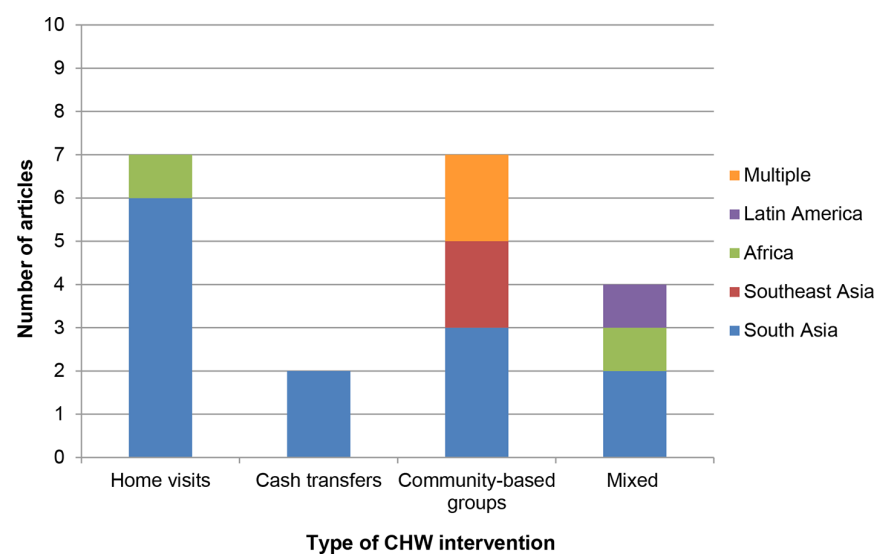

Figure 2 Number of articles reporting on each type of $\mathrm{CHW}$ intervention by region. $\mathrm{CHW}$, community health worker.

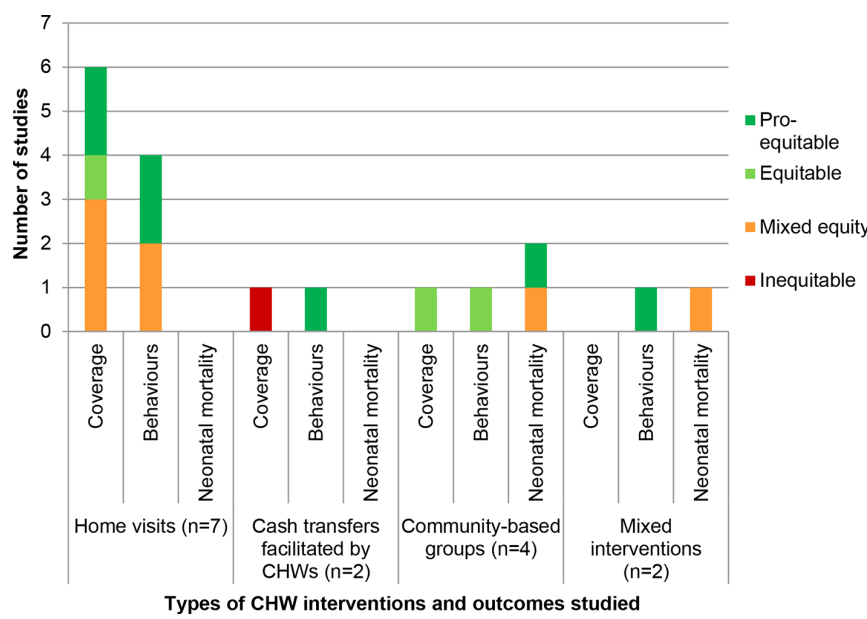

Figure 3 Number of studies reporting equity effects for different types of community interventions and outcomes. $\mathrm{CHW}$, community health worker.

community midwives. Some articles reported on the same interventions: four on participatory women's groups in Northeast India, ${ }^{25-28}$ three on participatory women's groups in $\mathrm{Nepal}^{262729}$ and two on local stakeholder group interventions in Vietnam. ${ }^{30} 31$

\section{Equity in coverage and effects on behaviours and mortality}

We categorised results from articles included in the review as follows: 'pro-equitable' meant that increases in coverage and/or intervention effects were greater among lower socioeconomic groups; 'equitable' meant increases in coverage or effects on outcomes were not significantly different between socioeconomic groups; 'mixed' described pro-equitable or equitable effects for some outcomes and/or dimensions of socioeconomic position but not others; 'inequitable' meant that coverage and/ or effects were greater among socioeconomic groups defined as higher compared with lower.

Out of the 15, nine quantitative articles reported on equity in coverage of CHW interventions, ${ }^{26} 28$ 32-38 two of which reported on the same trial (recorded once in figure 3). ${ }^{26}{ }^{28}$ Evidence on coverage was varied, with four studies reporting equitable or pro-equitable coverage (three articles on home visits, ${ }^{32} 3738$ one article reporting on seven women's group trials in Asia and Africa ${ }^{26}$ ), three reporting mixed coverage (all three on home visits ${ }^{33-35}$ ) and one article reporting inequitable coverage (cash transfers provided by $\mathrm{CHWs}^{36}$ ).

Eight of the 15 articles reported effects on maternal and newborn health-related behaviours by socioeconomic position. ${ }^{27} 283233$ 38-41 The majority (six) of these articles reported equitable or pro-equitable effects: two articles on home visits, ${ }^{32} 33$ two articles on participatory women's groups based on six randomised controlled trials in Asia and Africa ${ }^{27} 28$ (reported once in figure 3), one on cash transfers ${ }^{39}$ and one on a mixed intervention. ${ }^{40}$ Two studies on home visits reported mixed equity effects. ${ }^{3841}$ 
Two articles based on six randomised controlled trials of a women's group intervention reported pro-equitable effects on neonatal mortality across socioeconomic groups (recorded once in figure 3). ${ }^{27}$ One trial evaluating community stakeholder groups also reported a pro-poor improvement in NMR in intervention compared with control areas among wealth quintiles but not education or ethnicity groups. ${ }^{31}$ One study of a national community health programme (in figure 3 included under 'Mixed interventions') found that the density of CHWs in poor areas had no association with NMR, whereas in richer areas there was an association with higher neonatal mortality. ${ }^{42}$

Online supplementary table 5 presents detailed results from equity-related analyses in the 15 quantitative studies. We describe effects for each of these types of interventions below.

\section{Home visits}

Seven studies focused on home visits by CHWs: six in South Asia ${ }^{32} 3435373841$ and one in sub-Saharan Africa. ${ }^{33}$ Six of them reported on equity in intervention coverage. ${ }^{32-35} 3738$ Baqui et als moderate quality study in Uttar Pradesh, India found that coverage of at least one antenatal home visit improved from $10.7 \%$ to $51.4 \%$ and was more equitable in the intervention area over three years (change in Concentration Index (CoI) -0.172 (95\% CI -0.200 to 0.143$)$ ) compared with control area (change in CoI 0.058 (95\% CI 0.020 to 0.095 )) based on wealth quintiles. ${ }^{32} \mathrm{~A}$ moderate quality study on misoprostol distribution by CHWs in one district of Nepal reported greater proportionate gains in coverage among the poorest two quintiles, though this remained slightly higher among the highest wealth quintile $(p=0.049)$; there were no significant differences in coverage by literacy at endline. ${ }^{37}$ Conversely, a moderate quality study on home visits by CHWs (Anganwadi workers and Accredited Social Health Activists, ASHAs) in Bihar, India reported that CHWs' provision of immunisation was higher in more educated versus less educated households (OR 1.39 (95\% CI 1.05 to 1.82), $\mathrm{p}=0.02$ ), while their coverage of food supplements was greater among families in the lowest compared with highest wealth quintile (OR 0.87 (95\% CI 0.79 to 0.96 ), $\mathrm{p}=0.007) .{ }^{34}$ Seth $e t$ $a l$ s moderate quality study in Uttar Pradesh, India found that receipt of any ASHA contact was equitable based on pregnant women's caste, household wealth, religion or literacy ${ }^{38}$ Singh et al's cross-sectional study in one sub-district in rural Uttar Pradesh also found that the coverage of ASHAs' services for birth registration was higher among women with higher compared with lower socioeconomic class (OR 0.47, $\mathrm{p}=0.55$ ) and education (OR $0.32, \mathrm{p}=0.21$ ), and among Hindu compared with Muslim religious groups (OR 4.41, $\mathrm{p}=0.05$ ), though not different between caste groups. ${ }^{35}$ They also found that coverage of ASHA services for antenatal and postnatal care check-ups was not significantly inequitable across any dimension of socioeconomic position, though the study was of lower quality due to a small sample and no comparison area. One lower quality, repeat cross-sectional study in Malawi examined the effects of the government's Community-based Maternal and Newborn Care programme that trained lay CHWs called Health Surveillance Assistants (HSAs) to make home visits and counsel on birth preparedness, complication readiness and essential newborn care. ${ }^{33}$ They found that coverage of at least one antenatal home visit remained significantly higher among women in the highest $(42.8 \%)$ compared with lowest $(29.0 \%)$ wealth quartiles at endline in intervention districts $(\mathrm{CoI}$ 0.079 (95\% CI 0.022 to 0.170$)) .{ }^{33}$ Overall, two studies showed pro-equitable coverage, one reported equitable coverage, while three showed mixed equity in coverage (between some but not other socioeconomic groups).

Four articles reported the equity effects of CHW home visits on behaviours, with two having pro-equitable and two having mixed equitable effects. ${ }^{32} 3384$ A moderate quality, quasi-experimental study of an Integrated Nutrition and Health Programme in Uttar Pradesh, India found that receiving at least one home visit from any CHW was associated with improved equity between wealth quintiles for many MNH behaviours-including having at least one antenatal check-up (change in CoI -0.172 (95\% CI -0.200 to -0.143$)$ ), birth and emergency preparedness (change in CoI -0.095 (95\% CI -0.144 to -0.047 )), skilled birth attendance (change in CoI -0.095 (95\% CI -0.128 to $-0.063)$ ), at least one postnatal check-up (change in CoI -0.225 (95\% CI -0.289 to -0.161$)$ ) and clean cord care (change in CoI -0.106 (95\% CI -0.125 to -0.087$)$ ) - in intervention but not control districts. ${ }^{32}$ Health care utilisation became slightly more equitable between wealth quintiles but remained low. Similarly, a pre-post study of moderate quality in Bangladesh in which CHWs provided referrals for complications, financial support for delivery and counselling led to equitable improvements between the highest and lowest wealth quintiles after two years in receipt of four or more antenatal visits (change in CoI $-0.268(95 \%$ CI -0.368 to -0.168$)$ ), skilled birth attendance at home (change in CoI -0.094 (95\% CI -0.190 to $0.002)$ ) and delivery in public facilities (change in CoI $-0.228(95 \%$ CI -0.620 to 0.160$)){ }^{41}$ Seth $e$ e $a l$ 's moderate quality study in Uttar Pradesh reported equitable effects between wealth and literacy groups, but not by caste or religion. ${ }^{38}$ Having any ASHA contact was independently associated with increased odds of having four or more ANC visits among women who were non-Muslim (OR 1.85 (95\% CI 1.33 to 2.58 ) ) and of low wealth status (OR 2.24 (95\% CI 1.66 to 3.03)). Having any ASHA contact was independently associated with increased odds of having a facility delivery among women who were of low wealth status (OR 2.11 (95\% CI 1.71 to 2.60)) and not literate (OR 2.34 (95\% CI 1.83 to 3.02)) and to a lesser extent those who were literate (OR 1.33 (95\% CI 1.02 to 1.71)). Callaghan-Koru et $a l^{33}{ }^{33}$ lower quality study of Malawi's HSAs making home visits also found that, by endline, the proportion of women from lower compared with higher wealth quartiles increased equitably for 
institutional delivery (change in CoI -0.059 (95\% CI -0.098 to -0.020$)$ ) and breastfeeding within one hour after delivery (change in CoI -0.038 (95\% CI -0.073 to $-0.003)$ ). The proportion of infants bathed after six or more hours improved equitably (change in CoI -0.041 $(95 \%$ CI -0.095 to -0.014$)$ ) by endline.

In summary, in five South Asian settings and one African setting, coverage of home visits was most often equitable between wealth quintiles and sometimes education level. Three of four studies showed that CHW home visit interventions had equitable effects on behaviours like antenatal care, skilled birth attendance and homebased newborn care between wealth groups. No studies reported effects on neonatal mortality between socioeconomic groups.

\section{Conditional cash transfers}

One study from Nepal found moderately strong evidence that coverage of a cash incentive offered by CHWs for giving birth at government hospitals was higher among wealthier than poorer women, likely because the study found they delivered in facilities more often. ${ }^{36}$

With respect to the equity effects on maternal and newborn health-related behaviours, a cross-sectional study of moderate quality in Bangladesh provided evidence that after being recruited by a CHW to receive a voucher for maternal health services, the adjusted marginal effect was stronger for women in the poorest wealth quintile compared with the higher four quintiles receiving any antenatal check-ups $(43 \%(25 \%-61 \%)$ vs $22 \%(13 \%-30 \%))$, skilled delivery $(68 \%(55 \%-81 \%)$ vs $42 \%(33 \%-50 \%))$ and postnatal check-ups (25\% (13\%$38 \%)$ vs $17 \%(10 \%-25 \%)) .^{39}$

Based on the limited evidence, cash transfers had mixed equity in coverage and effects on behaviours for women of lower compared with higher socioeconomic positions in South Asia, while evidence on mortality effects was absent.

\section{Community-based group interventions}

Two articles reported on coverage of community-based group interventions by socioeconomic position from high-quality trials. ${ }^{26}$ The first of these articles, in Jharkhand and Odisha states, eastern India, reported that coverage improved from $11 \%$ and $15 \%$ to $59 \%$ and $52 \%$ among the most and less marginalised (in terms of literacy, poverty/asset ownership and caste status), respectively. ${ }^{28}$ The second article, based on seven cluster randomised controlled trials in Nepal, India, Bangladesh and Malawi, showed that women's group coverage was equitable, with slightly lower attendance among the most educated (OR 0.92 (95\% CI 0.44 to 1.93)) and wealthiest (OR 1.19 (95\% CI 0.62 to 2.29)) women in all sites. ${ }^{26}$

Two articles reported the effects of group interventions on maternal and newborn health behaviours by socioeconomic group, both of high quality. ${ }^{27}$ One article was based on a randomised trial of participatory women's groups in India, ${ }^{28}$ and another on a meta-analysis of six randomised trials of such groups in India, Nepal, Bangladesh and Malawi $^{27}$ (which included the trial reported in Houweling et al $2013^{28}$ ). These studies found that women's group interventions strongly improved hygiene, thermal care, breastfeeding and delayed bathing equitably between the less and more marginalised women (the latter included those who were illiterate, very poor, with little or no land and from a Scheduled Caste or Tribe). There were no systematic differences in the effects on these practices across socioeconomic groups. The intervention did not improve health care utilisation in any socioeconomic group. ${ }^{27} 28$

Three articles reported on the equity impact of groupbased interventions on neonatal mortality. ${ }^{27} 2831$ Two of these were, together, based on six of the women's group randomised controlled trails mentioned above. ${ }^{27} 28$ Houweling et als high-quality meta-analysis of six trials of women's groups found that the intervention led to a $63 \%$ decline $(95 \%$ CI $48 \%$ to $73 \%$ ) in the NMR among the most marginalised (illiterate and poorest wealth quintile), compared with a $35 \%$ decline $(95 \%$ CI $15 \%$ to $50 \%$ ) among the less marginalised. ${ }^{27} \mathrm{~A}$ highquality cluster randomised controlled trial in Vietnam studied the Neonatal Health-Knowledge Into Practice (NeoKIP) group interventions, in which local stakeholders (commune health staff, women's union members, lay health workers and local decision makers) discussed, developed and implemented strategies to improve maternal and newborn health. ${ }^{31}$ The intervention led to a $44 \%$ reduction (OR 0.56 (95\% CI 0.36 to $0.89)$ ) in neonatal mortality in the third intervention year. This effect was pro-equitable by wealth status, with a $69 \%$ reduction (OR 0.31 (95\% CI 0.15 to 0.66 ), $\mathrm{p}<0.001$ ) in neonatal mortality for poor households but no significant reduction for non-poor households. Conversely, there was a $50 \%(95 \%$ CI $0.28 \%$ to $0.90 \%)$ reduction in neonatal mortality among infants of women with at least primary education compared with an insignificant reduction among infants of women with less than primary education. The study found no differential effects between minority versus Kinh ethnic groups.

\section{Mixed interventions}

Two studies examined the effects of mixed CHW interventions involving both individual and group-based interventions as well as community skilled birth attendants (CSBAs) on equity in maternal and newborn health behaviours. ${ }^{40} 42$ The first was a moderate quality pre-post study of an integrated maternal health intervention in a rural sub-district of Bangladesh, which involved governmental and non-governmental partners to train CSBAs for six months in providing basic skilled delivery care at home, equip them with a clean delivery kit and link them to referral health facilities. ${ }^{40}$ They also developed Community Support Groups to host regular meetings, inform community members about the CSBA programme, and involve them in identifying and visiting pregnant women every month. The study found that in 
areas with lower compared with higher household wealth status and access to services, there were greater increases in the proportion of women having four or more antenatal checkups (OR 7.2 (95\% CI 3.6 to 14.3) vs 1.9 (95\% CI 1.1 to 3.3)) and delivery from a skilled provider (OR $4.0(95 \%$ CI 3.3 to 7.2$)$ vs 1.7 (95\% CI 1.3 to 2.3$)$ ) after three years of intervention. ${ }^{40}$

One study on Brazil's 'Single Health System' (Sistema Unico de Saude) found mixed results, as the presence of CHWs in poor areas where more than half of the population was below the poverty line was not associated with lower neonatal mortality, but there was a $1.25 \%$ reduction in neonatal mortality with every increase of one CHW per 1000 people overall. ${ }^{42}$ This study was of low quality, with an ecological design that did not account for individual-level characteristics or different CHW roles and training levels. ${ }^{42}$

\section{Intervention strategies and contextual processes}

The studies included in the review discussed intervention strategies and contextual processes underlying the equity effects of CHW interventions. We categorised these within four themes: CHW characteristics and intervention strategies; health care system; community processes and support; and political, socioeconomic and geographical processes.

\section{Community health worker characteristics and intervention strategies}

In moderate quality studies of home visits and participatory women's groups, CHWs' characteristics contributed to achieving equitable maternal and newborn health outcomes, including their residence, familiarity and ability to build relationships in the village, and degree of similarity in socioeconomic characteristics. According to a moderately strong qualitative study from Kenya, community midwives' local residence and presence in the social networks of women and families contributed to improved skilled birth attendance and protective homebased care practices across socioeconomic groups. ${ }^{43} \mathrm{~A}$ moderate quality qualitative study from Nepal also found that local residence of CHWs was linked to improvements in behavioural and mortality outcomes, especially among remote, low caste or poorer groups. ${ }^{29}$ A qualitative study of moderate quality in Haryana, India described how CHWs providing behaviour change communication during home visits, as well as support to access ambulance services and incentives, had increased the number of poor women delivering at free public facilities, where the MMR and NMR had also been reducing. ${ }^{44}$ One low quality study reported that when the female CHW and women she visited were of similar social status (most often by caste or religion), there was higher uptake of protective health practices. ${ }^{34}$ In a high-quality mixed-methods study in Pakistan, CHWs usually gave more attention to lower caste women with less 'social distance' from themselves, while their services were declined by higher castes. ${ }^{45}$ Conversely, a high-quality qualitative study from
Pakistan found that community midwives tended to be from wealthy families, and interacted less often with poorer women. ${ }^{46}$

Studies of moderate quality discussed how intervention strategies such as supportive supervision, job aids to provide regular feedback and reporting, and strong relationships with governmental or non-governmental partners providing technical support all contributed to equitable improvements in skilled birth attendance and essential newborn care practices by wealth status (community midwives in Kenya and home visits in India), as well as equitable improvements in neonatal mortality by wealth and literacy (women's groups in Nepal). ${ }^{29} 3243$ However, CHWs' ability to improve equitable coverage and effects were reduced by heavy workloads, low education levels or shortages in CHW recruitment. ${ }^{29} 324344$ In a low-quality study in India, CHWs' ability to improve equitable coverage and effects was also affected by the low ratio of CHWs to the population. ${ }^{34}$ Studies of high and moderate quality on participatory women's groups found that 'soft-targeting' heightened equitable coverage by proactively ensuring that those from lower socioeconomic groups could attend and benefit from meetings, holding meetings near the homes of poorer, more illiterate tribal families and at convenient times, ensuring people felt that membership was not exclusive, and developing accessible tools to use during meetings. 25262930

\section{Health care system}

Low health facility availability and quality seemed to widely underpin enduring inequities in coverage, care seeking and utilisation of services across contexts and intervention types. ${ }^{29} 303242$ Moderate quality studies found that even with cash transfers as incentives, high indirect costs were a barrier to institutional delivery in Nepal and Kenya. ${ }^{36} 43$ The provision of supplies, equipment and professional health care workers was generally poorer in villages with lower resources. ${ }^{32} 42-44$ Moderate quality qualitative studies from India and Vietnam found that poor training of nurses and doctors, as well as language barriers or discrimination against patients-which occurred more in ethnically diverse and remote communities-led to bad experiences in hospital, which in turn prevented the care seeking promoted by community-based groups. ${ }^{25}{ }^{30}$ Conversely, a low-quality study in Malawi stated that training government hospital staff likely reinforced the effects of home visits in improving newborn care practices, especially among the poorest who were more likely to use public rather than private services. ${ }^{33}$ Gupta et al's moderate quality study in Haryana, India found that improved availability of free medicines had supported CHWs' efforts to increase institutional deliveries among poor families, though some still opted to deliver at home due to fear of poor quality medicines and mistreatment from staff at government hospitals. ${ }^{44}$ 


\section{Community processes and support}

Studies of cash transfer and community-based group interventions discussed how the degree of community support played a role in hindering or enhancing equity effects. One moderate quality study suggested that families' growing acceptance of the Bangladesh voucher programme played a role in improving women's skilled birth attendance, antenatal and postnatal care among the lowest vs highest quintile groups. ${ }^{39}$ NeoKIP trial studies of high and moderate quality considered the role of community stakeholder groups in devising and implementing actions to address neonatal mortality, which led to reduced wealth-based inequities but increased educational inequities in neonatal mortality. ${ }^{3031}$ They discussed how the groups were largely comprised of poorer members who developed actions to enhance knowledge levels more often than change practices. Hence, they posited that more educated women may have been able to benefit most from knowledge-based approaches even if they were targeted to the poorest or minority groups. ${ }^{31}$ Among participatory women's groups, the social process of learning and acting together was found to reduce neonatal mortality most among those with lower socioeconomic positions. Community engagement by members (ie, talking to non-members) led to a diffusion of practices to prevent neonatal deaths among non-members. ${ }^{27}$ Many of their approaches to overcome contextual barriers were practical, such as removing transport or affordability constraints. ${ }^{25} 28$

\section{Political, economic and geographical processes}

A moderate quality study of a community midwife intervention in Bangladesh found that large distances to health facilities for rural and remote dwellers reduced pregnant women's receipt of misoprostol, care seeking and safe delivery. ${ }^{40}$ A moderate quality study on conditional cash transfer interventions facilitated by CHWs found that families still incurred out-of-pocket expenses for service fees, laboratory tests, drugs, transportation or gifts to providers, even after receiving the incentive, placing a disproportionate burden on the poorest. ${ }^{39}$ Studies in Brazil (low quality), India, Nepal and Vietnam (moderate quality) discussed how high levels of poverty, and lack of political will, infrastructure and resources countered the effects of home visit and group-based interventions on health care utilisation and neonatal mortality. ${ }^{25} 304244$ Limited health care utilisation after women's group interventions was attributed in part to geographical barriers to facilities in India and Bangladesh. ${ }^{25}$ Political instability and limited infrastructure and supplies, road closures and unsafe environments reduced care seeking in a women's group intervention in the Nepali context according to a moderate quality study. ${ }^{29}$ They highlighted challenges in addressing intractable political structures shaping socioeconomic inequities. ${ }^{29}$

\section{DISCUSSION}

This review found that there have been a small number of studies on the ability of CHW interventions to equitably achieve coverage and improve behavioural or mortality outcomes for maternal and newborn health between those of higher and lower socioeconomic position in LMICs. Coverage was pro-equitable in some but not all studies of CHW home visit and cash transfer interventions, and was equitable in women's group trials. Improved equity in behaviours and mortality was most evident in studies of CHWs providing home visits and facilitating participatory women's groups, particularly in home-based practices more than care seeking. This was more often observed between wealth quintiles and education than ethnicity or religion, and never examined by occupation groups. The results of this review have several implications for research and practice. First, researchers must increasingly consider the ways in which contextual factors, such as levels of poverty, political support or geographical barriers, interface with maternal and newborn health inequities between socioeconomic position groups. Second, there is a need to extend the study of equity from a focus on intervention coverage through to achieving equity in outcomes across the continuum of care. Finally, there is a need to better understand how socioeconomic inequities in maternal and newborn health may be addressed through better integration of facility-based and community-level efforts.

The review showed that $\mathrm{CHW}$ interventions have been most often linked to improved equity in the distribution of maternal and newborn health outcomes between wealth or education, and less often caste/tribe, religion or ethnicity groups. However, multiple dimensions of socioeconomic position and their role in shaping maternal and newborn health inequities are likely to be interlinked, as well as reinforced by political, socioeconomic and cultural factors. ${ }^{47-50}$ In this review, studies on each type of intervention showed that CHWs could change individuals' behaviours equitably. A few that examined women's group interventions' influence on wider community norms showed how income or education inequities commingled with community-level issues like social discrimination, but also that community engagement with village leaders helped change norms that had previously prevented poorer young women's attendance and optimal health practices. ${ }^{25} 263048$ Still, there is little evidence that CHW interventions can overcome the influence of structural determinants like low education, poverty, poor infrastructure or limited political will that arguably fuel existing socioeconomic inequities, and seemed to underlie poorer health care quality in all regions in this review. ${ }^{2530324347-50}$ Bishai et al' $\mathrm{s}^{51}$ analyses found that half of the reductions in the maternal and neonatal mortality across 146 LMICs between 1990 and 2010 were related to health sector-related improvements while the other half was attributable to improvements in non-health sectors, including economic growth, governance, education, infrastructure, water and sanitation. Future research employing both quantitative and qualitative methods should, therefore, investigate maternal and newborn health inequities in terms of multiple 
characteristics of socioeconomic position at once, and how the efforts of CHW interventions to address these inequities may be supported through investments in non-health sectors. ${ }^{5253}$

A second aspect of the review with research and policy implications is that most studies focused on equity in coverage and behaviours, yet there remains a paucity of evidence on equity impacts for maternal and neonatal mortality. While previous reviews found that there were effects of home visits or women's group interventions on mortality overall, they did not detect subgroup differences, and we found that only a few studies on participatory women's groups linked improved equity in coverage to equity in behaviours or mortality. ${ }^{25} 28$ This could relate to an inadequacy of conventional methodologies, or an implicit theoretical assumption that equity is achieved once everyone has access, by giving equal freedom of choice to enact healthy behaviours, but not also prioritising equitable realisation of health outcomes. ${ }^{12} 4954$ Future studies should focus on CHW interventions' equity effects across the continuum of care, particularly including mortality. ${ }^{55}$

Finally, while there is a working assumption that CHWs can redress widespread gaps in formal care in low-resource settings, this review underlines that linkages with the formal health care system are crucial not only in achieving effectiveness but also equity in maternal and newborn health outcomes. ${ }^{12}$ Past studies of community interventions' overall effectiveness for maternal and newborn health have found that both home-based care practices and care seeking have generally been improved, ${ }^{49} 56$ while our review showed that the latter was not achieved as equitably as the former. Many interventions relied heavily on demand generation as the main strategy, with a focus on mobilising people to use services. The challenges that some home visiting as well as community-based group interventions experienced for heightening care seeking equitably, particularly in more remote regions, calls into question the ability of CHWs to overcome supply-side health system barriers playing out across the 'time' dimension (prenatal to postnatal period) of the continuum of care, as well as the 'space dimension' between community-family care and facility-based clinical services. ${ }^{9} 12$ These contextual challenges underscore the urgent need to support CHWs' efforts by addressing, rather than compensating for, gaps in formal health services' accessibility, availability, quality and affordability. ${ }^{12} 48$ More multilevel analyses to consider how equity effects on maternal and newborn health outcomes are influenced by health system issues in different locales, like the degree that adequate provision of supplies, health personnel and delivery points are available or accessible, will likewise be needed. Implementation research would also be valuable to understand how stronger linkages between community interventions and facility-based clinical care may be able to reduce inequities. ${ }^{57}$
This mixed-methods review has some strengths and limitations. Our inclusion of both quantitative and qualitative studies helped to assess whether CHW intervention strategies have worked to reduce inequities in maternal and newborn health, as well as how and in what contexts they have worked. We also included studies of equity in interventions' coverage and their effects on equity in behaviours and mortality. However, by including a range of study designs, we were not able to use statistical methods to compare the quantitative results because the results often had little methodological comparability. Another limitation was that the initial analysis stage of coding the articles was not completed by multiple authors in tandem due to logistical constraints. We aimed to reduce the bias that this could have caused by ensuring that two authors interacted with the raw coded text to synthesise the results on what works, and having all authors discuss results in relation to the original texts in the process of developing themes on how and in what contexts. There may have been some publication bias favouring positive results, as equity analysis may constitute a step beyond effectiveness studies that researchers would not take if found ineffective. Still, some studies reported negative or mixed effects on equity. There was limited programme and contextual detail in many studies. The grey literature could have provided more information about this, but would have yielded an unwieldy amount of information given the necessarily wide inclusion criteria for study designs. However, the use of narrative synthesis helped to explore intervention strategies and contextual processes.

\section{CONCLUSION}

This review aimed to address the crucial need to understand what evidence exists on CHW interventions' effects on equity in maternal and newborn health, and particularly the intervention strategies and contextual processes shaping them. Though evidence was somewhat limited, it showed that CHW interventions have equitably improved maternal and newborn health service utilisation or homebased practices, and even reduced inequities in neonatal mortality in some contexts. There is still much to be done to consider multiple socioeconomic dimensions contributing to health inequities and their roots in contextual processes, whether equitable coverage follows through to equity in behaviours and especially mortality, and the effects of integrating CHW activities with wider health system strengthening activities. The review provides hope that when CHW interventions are supported by purposive soft-targeting and linkages with community and health system actors, they can play a key role in improving equity in maternal and newborn health.

Contributors AKB and AP planned and conducted the search and synthesis of findings with input from TAJH, and all authors worked on analysis and reporting of results.

Funding TAJH was supported by a grant from the Erasmus Trustfonds, Erasmus University Rotterdam, The Netherlands. 
Disclaimer The funder had no role in study design, data collection, analysis, interpretation, decision to publish or preparation of the manuscript.

Competing interests None declared.

Patient consent for publication Not required.

Provenance and peer review Not commissioned; externally peer reviewed.

Data sharing statement There are no data in this work.

Open access This is an open access article distributed in accordance with the Creative Commons Attribution Non Commercial (CC BY-NC 4.0) license, which permits others to distribute, remix, adapt, build upon this work non-commercially, and license their derivative works on different terms, provided the original work is properly cited, appropriate credit is given, any changes made indicated, and the use is non-commercial. See: http://creativecommons.org/licenses/by-nc/4.0/.

\section{REFERENCES}

1. United Nations. Sustainable development goal 3: ensure healthy lives and promote well-being for all at all ages. Secondary sustainable development goal 3: ensure healthy lives and promote well-being for all at all ages, 2018. Available: https://sustainabled evelopment.un.org/sdg3

2. Lawn JE, Blencowe H, Oza S, et al. Every newborn: progress, priorities, and potential beyond survival. Lancet 2014;384:189-205.

3. UNICEF. Maternal and Newborn Health Disparities - India. Geneva UNICEF; 2016: 1-6.

4. Tulenko K, Møgedal S, Afzal MM, et al. Community health workers for universal health-care coverage: from fragmentation to synergy. Bull World Health Organ 2013;91:847-52.

5. United Nations. Sustainable Development Goals. Secondary sustainable development goals, 2018. Available: https://www.un.org/ sustainabledevelopment/health/

6. Olaniran A, Smith $H$, Unkels $R$, et al. Who is a community health worker? - a systematic review of definitions. Glob Health Action 2017:10:1272223-13

7. World Health Organization. Community health workers: a strategy to ensure access to primary health care services. Cairo: Regional Office for the Eastern Mediterranean, 2016: 1-37.

8. Lehmann U, Sanders D. Community health workers: what do we know about them? The state of the evidence on programmes, activities, costs and impact on health outcomes of using community health workers. Geneva World Health Organization; 2007: 1-42.

9. Lassi ZS, Haider BA, Bhutta ZA. Community-based intervention packages for reducing maternal and neonatal morbidity and mortality and improving neonatal outcomes. Cochrane Database Syst Rev 2010;(11):CD007754.

10. Yuan $B$, Målqvist $M$, Trygg $N$, et al. What interventions are effective on reducing inequalities in maternal and child health in low- and middle-income settings? A systematic review. BMC Public Health 2014;14:1-14

11. McCollum R, Gomez W, Theobald S, et al. How equitable are community health worker programmes and which programme features influence equity of community health worker services? A systematic review. BMC Public Health 2016;16.

12. Schleiff M, Kumapley R, Freeman PA, et al. Comprehensive review of the evidence regarding the effectiveness of community-based primary health care in improving maternal, neonatal and child health 5. equity effects for neonates and children. J Glob Health 2017;7.

13. Perry M, Becerra F, Kavanagh J, et al. Community-based interventions for improving maternal health and for reducing maternal health inequalities in high-income countries: a systematic map of research. Global Health 2015;10.

14. Östlin P, Schrecker T, Sadana R, et al. Priorities for research on equity and health: towards an equity-focused health research agenda. PLoS Med 2011;8:e1001115.

15. Petticrew M, Roberts H. Systematic Reviews in the Social Sciences: a Practical Guide. Blackwell Publishing: Oxford, 2006.

16. Pawson R, Greenhalgh T, Harvey G, et al. Realist review--a new method of systematic review designed for complex policy interventions. J Health Serv Res Policy 2005;10 Suppl 1:21-34.

17. Shepperd S, Lewin S, Straus S, et al. Can we systematically review studies that evaluate complex interventions? PLoS Med 2009;6:e1000086.

18. Welch V, Petticrew M, Tugwell P, et al. PRISMA-Equity 2012 extension: reporting guidelines for systematic reviews with a focus on health equity. PLoS Med 2012;9:e1001333.
19. Welch V, Petticrew M, Petkovic J, et al. Extending the PRISMA statement to equity-focused systematic reviews (PRISMA-E 2012): explanation and elaboration. Int J Equity Health 2015;14:1-23.

20. Schiffman J, Darmstadt GL, Agarwal S, et al. Communitybased intervention packages for improving perinatal health in developing countries: a review of the evidence. Semin Perinatol 2010;34:462-76.

21. Kok MC, Dieleman M, Taegtmeyer M, et al. Which intervention design factors influence performance of community health workers in low- and middle-income countries? A systematic review. Health Policy Plan 2015;30:1207-27

22. Upadhyay RP, Chinnakali $\mathrm{P}$, Odukoya $\mathrm{O}$, et al. High neonata mortality rates in rural India: what options to explore? ISRN Pediatr 2012;2012:1-10.

23. O'Neill J, Tabish $\mathrm{H}$, Welch $\mathrm{V}$, et al. Applying an equity lens to interventions: using progress ensures consideration of socially stratifying factors to illuminate inequities in health. J Clin Epidemiol 2014;67:56-64.

24. Ueffing E, Tugwell P, Welch V, et al. Equity checklist for systematic review authors, 2012. Available: http://equity.cochrane.org/ sites/equity.cochrane.org/files/uploads/EquityChecklist2012.pdf [Accessed 26 May 2016].

25. Rath S, Nair N, Tripathy PK, et al. Explaining the impact of a women's group led community mobilisation intervention on maternal and newborn health outcomes: the Ekjut trial process evaluation. BMC Int Health Hum Rights 2010;10:1-13.

26. Houweling TAJ, Morrison J, Alcock G, et al. Reaching the poor with health interventions: programme-incidence analysis of seven randomised trials of women's groups to reduce newborn mortality in Asia and Africa. J Epidemiol Community Health 2016;70:31-41.

27. Houweling TAJ, Looman CWN, Azad K, et al. The equity impact of community women's groups to reduce neonatal mortality: a meta-analysis of four cluster randomized trials. Int $\mathrm{J}$ Epidemiol 2019;48:168-82.

28. Houweling TAJ, Tripathy $P$, Nair N, et al. The equity impact of participatory women's groups to reduce neonatal mortality in India: secondary analysis of a cluster-randomised trial. Int J Epidemiol 2013;42:520-32

29. Morrison J, Thapa R, Hartley S, et al. Understanding how women's groups improve maternal and newborn health in Makwanpur, Nepal: a qualitative study. Int Health 2010;2:25-35.

30. Duong DM, Bergström A, Wallin L, et al. Exploring the influence of context in a community-based facilitation intervention focusing on neonatal health and survival in Vietnam: a qualitative study. BMC Public Health 2015;15:1-9.

31. Målqvist M, Hoa DPT, Persson Lars-Åke, et al. Effect of facilitation of local Stakeholder groups on equity in neonatal survival; results from the NeoKIP trial in northern Vietnam. PLoS One 2016;10:e0145510.

32. Baqui $A H$, Rosecrans $A M$, Williams EK, et al. NGO facilitation of a government community-based maternal and neonatal health programme in rural India: Improvements in equity. Health Policy Plan 2008;23:234-43

33. Callaghan-Koru JA, Nonyane BAS, Guenther T, et al. Contribution of community-based newborn health promotion to reducing inequities in healthy newborn care practices and knowledge: evidence of improvement from a three-district pilot program in Malawi. BMC Public Health 2013;13.

34. Kosec K, Avula R, Holtemeyer B, et al. Predictors of essential health and nutrition service delivery in Bihar, India: results from household and frontline worker surveys. Glob Health Sci Pract 2015;3:255-73.

35. Singh MK, Singh J, Ahmad N, et al. Factors influencing utilization of ASHA services under NRHM in relation to maternal health in rural Lucknow. Indian J Community Med 2010;35:414-9.

36. Powell-Jackson T, Neupane BD, Tiwari S, et al. The impact of Nepal's National incentive programme to promote safe delivery in the District of Makwanpur. Adv Health Econ Health Serv Res 2009;21:221-49.

37. Rajbhandari S, Hodgins S, Sanghvi H, et al. Expanding uterotonic protection following childbirth through community-based distribution of misoprostol: Operations research study in Nepal. Int J Gynaecol Obstet 2010;108:282-8

38. Seth A, Tomar S, Singh K, et al. Differential effects of community health worker visits across social and economic groups in Uttar Pradesh, India: a link between social inequities and health disparities. Int J Equity Health 2017;16.

39. Nguyen HTH, Hatt L, Islam M, et al. Encouraging maternal health service utilization: an evaluation of the Bangladesh voucher program. Soc Sci Med 2012;74:989-96.

40. Huq NL, Ahmed A, Haque Nal, et al. Effect of an integrated materna health intervention on skilled provider's care for maternal health 
in remote rural areas of Bangladesh: a pre and post study. BMC Pregnancy Childbirth 2015;15:1-15.

41. Quayyum Z, Khan MNU, Quayyum T, et al. "Can community level interventions have an impact on equity and utilization of maternal health care" - Evidence from rural Bangladesh. Int J Equity Health 2013;12:22-12.

42. Sousa A, Dal Poz MR, Boschi-Pinto C. Reducing inequities in neonatal mortality through adequate supply of health workers: evidence from newborn health in Brazil. PLOS ONE 2013;8:e74772.

43. Mannah MT, Warren C, Kuria S, et al. Opportunities and challenges in implementing community based skilled birth attendance strategy in Kenya. BMC Pregnancy Childbirth 2014;14:1-12.

44. Gupta M, Bosma H, Angeli F, et al. Impact of a Multi-Strategy community intervention to reduce maternal and child health inequalities in India: a qualitative study in Haryana. PLoS One 2017;12.

45. Mumtaz Z, Salway S, Nykiforuk C, et al. The role of social geography on lady health workers' mobility and effectiveness in Pakistan. Soc Sci Med 2013;91:48-57.

46. Mumtaz Z, Levay A, Bhatti A, et al. Good on paper: the gap between programme theory and real-world context in Pakistan's community midwife programme. BJOG: Int J Obstet Gy 2015;122:249-58.

47. Bauer GR. Incorporating intersectionality theory into population health research methodology: challenges and the potential to advance health equity. Soc Sci Med 2014;110:10-17.

48. Starfield B. Pathways of influence on equity in health. Soc Sci Med 2007;64:1355-62.

49. Yuan $B$, Målqvist $M$, Trygg $\mathrm{N}$, et al. What interventions are effective on reducing inequalities in maternal and child health in low- and middle-income settings? A systematic review. BMC Public Health 2014;14.

50. O'Campo P, Dunn JR, eds. Rethinking Social Epidemiology: Towards a Science of Change. New York: Springer Science+Business Media B.V., 2012.

51. Bishai DM, Cohen R, Alfonso YN, et al. Factors contributing to maternal and child mortality reductions in 146 low- and middle-income countries between 1990 and 2010. PLoS One 2016;11:e0144908.

52. Bhutta ZA, Das JK, Bahl R, et al. Can available interventions end preventable deaths in mothers, newborn babies, and stillbirths, and at what cost? The Lancet 2014;384:347-70.

53. Solar O, Irwin A. A conceptual framework for action on the social determinants of health. social determinants of health discussion paper 2 (policy and practice). Geneva World Health Organization; 2010: 1-79.

54. Sen A. The Idea of Justice. Cambridge, Massachusetts: Harvard University Press, 2009

55. Kikuchi K, Ansah EK, Okawa S, et al. Effective linkages of continuum of care for improving neonatal, perinatal, and maternal mortality: a systematic review and meta-analysis. PLoS One 2015;10.

56. Kok MC, Kane SS, Tulloch O, et al. How does context influence performance of community health workers in low- and middleincome countries? Evidence from the literature. Health Res Policy Syst 2015;13

57. Theobald S, Brandes N, Gyapong M, et al. Implementation research: new imperatives and opportunities in global health. Lancet 2018;392:2214-28. 